\title{
Eating Online Discourses: Rhetorics on Food Consumption in Contemporary Bicocca (Milan, Italy)
}

\author{
Luca Rimoldi \\ PhD in Anthropology \\ Università degli Studi di Milano-Bicocca \\ Email rimoldi.luca@gmail.com
}

\section{Doi:10.5901/jesr.2014.v4n1p469}

\begin{abstract}
This article sets out to analyse from both anthropological and comparative perspectives two trattorie -the Tuscan Trattoria da Aldo and the Old Trattoria Arlati- in Bicocca, a post-industrial Milanese neighbourhood. In order to best consider food consumption rhetorics in these historical places, I am using ethnographic observation, employing as sources web portal online reviews from sites such as Tripadvisor. it, 2spaghi.it, and Yelp.it, blog contents, and the restaurants' own official websites. Here, the consumption of food plays an important role in revealing how the so-called "new" inhabitants of this area perceive, interpret, and excite new discourse on the social memory of the area itself.
\end{abstract}

Keywords: Anthropology, Food Consumption, Virtual Ethnography, Memory, Bicocca neighbourhood

\section{Introduzione}

Questo articolo è frutto di alcune riflessioni connaturate alla ricerca etnografica condotta tra il 2008 e il 2011 a Milano nel quartiere Bicocca. I miei interessi vertevano sulla costruzione di una serie di discorsi sulla memoria operaia attraverso le narrazioni di un gruppo di ex-lavoratori della Pirelli-Bicocca (Rimoldi, 2012). Dall'inizio del Novecento fino agli anni '70 il quartiere Bicocca ha ospitato la sede delle Industrie Pirelli. Negli anni '90 la riconversione ha prodotto un intero nuovo quartiere, dando luogo a una delle più grandi trasformazioni urbane d'Europa. Quella della Pirelli-Bicocca era un'area industriale di $750.000 \mathrm{mq}$ composta da stabilimenti che, fino agli anni '80, occupavano oltre 15.000 dipendenti; oggi, invece, i vecchi capannoni industriali hanno lasciato spazio al moderno campus dell'Università degli Studi di MilanoBicocca, alla sede del Consiglio Nazionale delle Ricerche (CNR), ai nuovi centri direzionali della Pirelli, della Siemens e della Deutsche Bank, al Teatro degli Arcimboldi e al Bicocca Village, un centro commerciale con cinema multisala. Se, come sostengono gli studi di antropologia urbana, vivere in un particolare spazio percepito come delimitato fa emergere delle caratteristiche diverse da quelle che si manifestano in altri luoghi (Hannerz, 1992), allora prendere in considerazione le ricadute sociali che le trasformazioni del quartiere hanno avuto e continuano ad avere nella definizione delle pratiche della quotidianità dei suoi abitanti appare come qualcosa di particolarmente rilevante dal punto di vista antropologico.

Il cambiamento della vocazione del quartiere, da industriale a terziaria, ha senza dubbio portato a una ridefinizione della composizione sociale dei suoi abitanti. Sebbene non manchino edifici residenziali, nuovi o in corso di costruzione, il quartiere Bicocca appare, agli occhi di chi lo visita oggi, come un luogo che intercetta enormi flussi di persone che popolano nelle ore diurne le strade e gli edifici pressoché deserti nelle ore serali. Se fino agli anni '80 gli operai della Pirelli-Bicocca e delle altre fabbriche della zona erano da considerarsi come gli abitanti del quartiere, ora gli studenti, i lavoratori dell'Università e delle aziende presenti sul territorio, così come le persone che rispondono all'offerta di intrattenimento proposta dal quartiere sono a mio avviso considerabili come "nuovi abitanti". In questa prospettiva, il consumo del cibo -pratica sociale che caratterizza la quotidianità del quartiere- sembra essere una chiave di lettura particolarmente interessante per mostrare come i "nuovi abitanti" del quartiere intercettino e reinterpretino i discorsi sulla memoria del quartiere stesso.

In un recente articolo (Rimoldi, 2013) ho focalizzato la mia attenzione sul legame che, nei mesi della mia ricerca, si è andato a creare tra il consumo del cibo e la costruzione dell'incontro etnografico tra me e i miei interlocutori parlandone in termini di "pretesto sociale". Ciò che è emerso è l'articolazione di una serie di discorsi in cui al consumo del cibo e alle pratiche di commensalità veniva associata una più ampia prospettiva legata alla narrazione delle lotte del 
'68 in Bicocca, al controllo dei luoghi della commensalità ${ }^{1}$, in altre parole, al valore simbolico e identitario del cibo come catalizzatore della memoria individuale e collettiva; ho pertanto iniziato a considerare il consumo del cibo come un'arena in cui si costruisce la complessità dei discorsi sulla memoria.

In questo articolo mi interrogo sulle retoriche relative al consumo del cibo e sui significati che a esse vengono attribuite da una serie di discorsi degli utenti di alcune comunità online, i membri delle quali hanno tessuto, e continuano a tessere, la memoria del quartiere in luoghi diversi dal quartiere stesso. Nel caso etnografico che presento, mostro proprio come le retoriche sul consumo del cibo veicolate dalla rete attribuiscano una serie di significati che vanno a ripensare gli spazi del quartiere Bicocca di Milano alimentandone così la memoria. Non è solo attraverso le interazioni che le persone hanno all'interno di una città o di un quartiere che vengono costruite le relazioni che in essi prendono vita. Come ha messo in luce Pierre Lévy, infatti, il virtuale si sovrappone, come spazio antropologico, agli spazi più classicamente familiari agli antropologi (1997). Internet, in questo senso, ha un ruolo centrale in quella che potrei definire come "un'esportazione" in una realtà virtuale delle interazioni tra le persone, utenti, che coi i loro discorsi attribuiscono ulteriori significati ai luoghi e contribuiscono a tesserne la memoria².

La letteratura antropologica ha messo più volte in discussione se internet fosse un campo di studi adatto agli antropologi e se l'etnografia fosse un metodo adatto per analizzare dei fenomeni parasociali anziché reali (Calhoun, 1991; Baulieu, 2004). Sicuramente la mancanza di un contatto faccia a faccia dell'antropologo con i suoi interlocutori e le modalità di comunicazione degli utenti attraverso il web possono rappresentare una sfida per una metodologia che cerca di indagare le forme di socialità che il consumo del cibo crea. Tuttavia, seguendo Hine, «Per l'etnografo la questione non è quella di portare un qualche criterio esterno per giudicare se sia sicuro credere alle parole che gli informatori dicono, quanto piuttosto quello di arrivare a capire cosa gli informatori giudicano come autentico» (Hine, 2000: 49). Dunque, ciò che viene percepito come autentico nell'analisi dei discorsi online, così come negli incontri etnografici è negoziato in modo circostanziale.

Per portare avanti la mia argomentazione mi sono basato sulle parole che gli utenti di alcuni portali web (Tripadvisor.it, 2Spaghi.it. e Yelp.it3) hanno scritto negli ultimi anni per recensire due luoghi storici della commensalità del quartiere Bicocca oltre che sul contenuto di alcuni blog e dei siti ufficiali della Trattoria Toscana da Aldo e della Trattoria Arlati. Gli utenti di questo tipo di comunità virtuali non si limitano a esprimere giudizi di merito rispetto al rapporto tra la qualità del servizio offerto dai ristoranti ma spesso tendono a condividere l'esperienza che hanno vissuto in un determinato luogo anche attraverso lunghe descrizioni del locale, degli altri avventori incontrati sul posto e delle interazioni con il personale. In questo senso, dunque, l'interazione informata tra gli utenti crea spazi virtuali che non sono da considerarsi come distinti e separati da quelli reali (Carter, 2005) e, pertanto, la loro interazione e i loro discorsi si rivelano essere delle fonti etnografiche particolarmente significative (Hine, 2000; Murphy, 2011) e in grado di mostrare l'articolazione dei discorsi sulla memoria nella contemporaneità del quartiere Bicocca. D'altro canto, i siti ufficiali e i commenti dei gestori delle due trattorie, attraverso le risposte alle recensioni lasciate dagli utenti, mettono bene in evidenza gli intenti legati a un certo tipo di autorappresentazione digitale e la loro ricaduta nella quotidianità del quartiere. In questo articolo presento un'analisi comparativa di due luoghi della commensalità del quartiere. Pur essendo entrambi legati alla storia delle industrie Pirelli e a quelle dei suoi operai, la Trattoria Toscana da Aldo e la Trattoria Arlati hanno percorso cammini molto diversi dal punto di vista delle retoriche del consumo del cibo. Entrambe, attraverso la socialità che creano, contribuiscono a veicolare differenti rappresentazioni di un medesimo luogo. Dal momento che, come vedremo, entrambe le trattorie si autorappresentano online proprio come luoghi di memoria reclamando l'autenticità dei discorsi che portano avanti, l'articolo indaga come tali rappresentazioni vengano accettate o messe in discussione dai recensori online.

\footnotetext{
${ }^{1}$ Come mi hanno riferito, tra gli altri, Alberto e Luciano Stipiti, attuali proprietari della trattoria da Aldo, prima dellintroduzione dello Statuto dei Lavoratori del 1970 non era permesso ai funzionari sindacali l'ingresso in fabbrica e, pertanto, gli incontri con delegati e rappresentanti di reparto avvenivano proprio in alcuni luoghi della commensalità che sorgevano introno all'area della Pirelli Bicocca.

2 Faccio riferimento all'idea di memoria che emerge dagli studi di antropologia e storia orale. Per approfondire, si vedano i testi di Fabietti e Matera (2000), di Portelli (1985; 2007) e di Sutton (2011) e gli articoli di Dei (2004) e Berliner (2005).

${ }^{3}$ Ho scelto di prendere in considerazione 2Spaghi le versioni italiane di Tripavisor e Yelp per la loro popolarità sul web. Tripadvisor è il sito di viaggi più grande al mondo, nella sezione "ristoranti" si contano circa 1.100 .000 locali sparsi in 24.000 città del mondo. Per accedere a tutti e tre i siti occorre effettuare una registrazione dei propri dati personali ma è anche possibile accedervi attraverso il proprio profilo Facebook.
} 


\section{Consumo del Cibo e Memoria Operaia nella Trattoria Toscana da Aldo}

La Trattoria Toscana da Aldo, sita in Viale Sarca 187, è stata aperta nel 1950 da Edoardo Stipiti (detto Aldo) e da sua moglie Elsa, entrambi originari di Ponte Buggianese (Pistoia) ed è ora gestita dai figli della coppia: Luciano (1948) e Alberto (1962). Vito Basilico (1926), uno dei protagonisti delle lotte operaie alla Pirelli Bicocca e uno dei miei interlocutori principali, ricorda che quando Aldo aveva appena aperto il suo locale si mangiavano solamente polenta e pesce fritto; il lavoro in cucina iniziava alle 5 del mattino dal momento che già alle 6 il locale si riempiva degli operai che smontavano dal turno di notte e si andava avanti fino a mezzanotte, dopo aver servito i turnisti delle 22. Luciano e Alberto Stipiti mi hanno raccontato che inizialmente il locale era una rosticceria e occupava circa la metà della superficie che occupa ora; i loro genitori avevano col tempo ampliato il menù e gli operai e gli impiegati della Pirelli-Bicocca, dopo aver ordinato il cibo, andavano a consumarlo nella vicina Casa del Popolo, a pochi passi di distanza seguendo viale Sarca in direzione sud. Poco prima dell'inizio degli anni '60 il locale inizia ad assumere l'aspetto che conserva ancora oggi: vengono collocati i primi tavolini e i clienti iniziano a consumare il cibo all'interno del locale. Come si legge nel blog ufficiale della trattoria:
«In viale Sarca 187 a Milano (zona Bicocca) c'è una trattoria storica: la Trattoria Toscana da Aldo! Siamo appunto in viale Sarca proprio affianco al numero 187 a Milano in zona Bicocca praticamente di fronte alle Università. Ve 10 assicuro: una trattoria così stile vecchia Milano è una cosa rara ormai. L'ambiente è colmo di simboli e il clima di classica trattoria è ovunque, nei libri, nei quadri, negli oggetti... Cucina casereccia e vino rosso a volontà. II menù cambia giornalmente con piatti tipici e anche alla moda! Insomma menù completo con:
- primo, secondo, contorno
- vino rosso o acqua a volontà
- caffè
- sambuca o ammazzacaffè in genere
12 euro
L'ultima sambuca...magari al bancone.
Vedete? Abbiamo degli amici che ve li sognate. E scrivono di noi anche sui quotidiani. E abbiamo anche ricevuto delle onorificenze. Quindi... a Voi la scelta!»s.

Già dalla presentazione che Luciano e Alberto Stipiti propongono della trattoria nel loro blog ufficiale emergono una serie di specificità del luogo stesso che cercherò di analizzare attraverso l'uso di altre recensioni comparse online. In primo luogo viene esplicitata la precisa collocazione del locale e la vicinanza con gli edifici dell'Università di MilanoBicocca, questo suggerisce che la clientela abituale della trattoria sia costituita principalmente da studenti. Come lo stesso Luciano ha dichiarato in un'intervista pubblicata da "II Giorno" (6 maggio 2007) a proposito della sua clientela degli ultimi anni:

«Niente più operai (...) da quando hanno spostato la portineria della Pirelli e niente più camionisti da quando la ristrutturazione di carreggiate e marciapiedi ha reso più difficile la sosta. Rimpiango un po' quei tempi, c'era sempre movimento e il clima era diverso. Entravano e mangiavano quello che c'era. Ora invece, gli studenti, arrivano in cinque e mangiano in due. (...) C'è anche da dire (...) che alcuni non se lo possono permettere e non vogliono pesare troppo sulle spalle dei genitori. Quando me ne accorgo propongo un patto: il pranzo in cambio di una mano nei giorni successivi» (Luciano Stipiti).

Durante la lunga frequentazione di questa trattoria mi è più volte capitato di incontrare, insieme agli ex-lavoratori delle Industrie Pirelli con i quali andavo a pranzo da Aldo per motivi di ricerca, alcuni studenti e impiegati dell'Università. I| 2 luglio del 2013, l'utente di Tripadivsor.com Richter S sembra confermare ciò che ho potuto osservare:

"Comoda trattoria per gli studenti della vicina università che hanno corsi al pomeriggio e vogliono provare di tanto in tanto una alternativa alla mensa. La qualità dei piatti è discreta e in proporzione i prezzi sono competitivi, considerando anche vino e caffè inclusi. Ambiente molto rustico; il proprietario è un soggetto particolare. Visitata solo una volta con un collega, ci tornerei volentieri per sapere se anche oggi è come allora». 5

Dopo aver sottolineato la posizione geografica, l'incipit della presentazione della Trattoria Toscana da Aldo

\footnotetext{
4 http://www.trattoriatoscanadaaldo.restaurant.point.in.citylandia.org (ultimo accesso: 30 ottobre 2013).

5 Utente Richter (2 luglio 2013) http://www.tripadvisor.it/Restaurant_Review-g187849-d2017514-Reviews-Trattoria_Toscana_Da_AldoMilan_Lombardy.html (ultimo accesso: 30 ottobre 2013). 
sembra spostarsi su un livello di affermazione del senso che si vorrebbe attribuire al luogo stesso: un luogo "autentico", unico nel suo genere, difficilmente riproducibile e, pertanto, raro; in questo senso ciò che viene rappresentato come autentico trova nella storicità ${ }^{6}$ del locale la sua manifestazione più evidente. In secondo luogo, infatti, viene posto l'accento proprio sulla storicità del locale e sul fatto che al suo interno siano presenti simboli il cui significato rimanda all'idea di "classica trattoria". A tale proposito si legge nella recensione di Gianluca L su Yelp:

\begin{abstract}
«La Trattoria Toscana da Aldo trasuda storia da tutte le pareti. Qua un tempo si nutrivano gli operai, ora i frequentatori sono generalmente gli studenti della Bicocca. I ritmi frenetici di Milano hanno costretto il mitico gestore ad adeguarsi ai tempi ed ora vendono anche panini e piadine. Ma questa trattoria, per godersela, va vissuta sedendosi. Sul muro si scorge una vecchia foto con la scritta "E' un momentaccio!". I tempi allora non sono tanto cambiatil! Qua pasteggiava anche Sergio Cofferati. La trattoria ha vissuto gli anni delle lotte e gli anni del disimpegno. Ora tutte quelle scritte e i Che Guevara sul muro paiono un po' come monumenti ai caduti. Per il resto un ottimo posticino dove mangiare ed essere maltrattati dal buon gestore. Difficile tornare a lezione dopo un lauto pasto accompagnato dal vinello della casas. ${ }^{7}$
\end{abstract}

Inoltre, non viene prestata particolare attenzione alla costruzione di un discorso sul cibo regionale. Pur avendo nel suo stesso nome un locativo esplicito, la cucina di Aldo non presenta piatti che fanno riferimento a una cucina "tradizionale" ma, piuttosto, a una cucina "casereccia". Pur avendo pranzato molte volte in questa trattoria non ricordo di aver mai ordinato un piatto particolare; i clienti abituali, infatti, dopo essersi seduti a un tavolo qualunque e aver salutato Luciano, Alberto e gli altri avventori, si siedono e aspettano che sia uno dei due fratelli Stipiti a portare loro ciò che c'è in cucina: solitamente un primo e un secondo.

"Da "Aldo" potete trovare piatti milanesi e toscani in un'atmosfera che potremmo definire "comunista 100\%" :). Ci sono 2 primi subito pronti (pasta con sughi piccanti o piccantissimi, consiglio l'amatriciana), e molti secondi di carne, saporiti, speziati e pepati! Si mangia in compagnia e su tavolate lunghe ma spaziose, quindi niente tavolini larghi $30 \mathrm{~cm}$ in cui si mangia con i gomiti sulle ginocchia; vino e acqua sono in perfetta condivisione comunista (le posate no), e il costo è ridicolmente basso per Milano. Ottimi i formaggi, taleggio o gorgonzola o pochi altri, ma veramente squisiti; il dolce invece c'è solo su prenotazione (ossia mai)».9

In generale, gli utenti delle comunità virtuali che scrivono recensioni sulla Trattoria Toscana da Aldo sottolineano l'appartenenza e l'inclusione del locale stesso ad un passato mitico del comunismo che vede il Fidel Castro della rivoluzione cubana e Che Guevara come icone ma che ha poco in comune con la tensione politica della sinistra italiana contemporanea. Per certi versi, dunque, si ritrova quel richiamo alla dimensione del «post-politico» (Esposito, 1988) che inquadra la sinistra come uno spazio (ricordato) ideale e morale. L'appartenenza di senso più che di partito sembra dunque configurare la Trattoria Toscana da Aldo come un luogo storico della commensalità. Utilizzando le recensioni apparse online come fonti etnografiche su cui riflettere, posso affermare che le rappresentazioni della Trattoria Toscana da Aldo non si legano tanto a discorsi relativi al cibo attraverso la presentazione di piatti regionali, tipici o particolarmente ricercati. Questa trattoria fabbrica una serie di retoriche relative al consumo del cibo e alla commensalità che esso crea sotto l'egida di una forte appartenenza politica rappresentata tanto dai simboli quanto dai clienti abituali del locale. Dal momento che questi ultimi sono perlopiù ex-lavoratori della Pirelli-Bicocca e delle fabbriche che sorgevano nelle vicinanze, ecco che allora l'immagine che viene creata dal locale si lega alla memoria del quartiere stesso a ciò che il quartiere era, un quartiere operaio, e si collega a ciò che è oggi, un quartiere universitario popolato nelle ore del giorno dai più di 30.000 studenti dell'Università.

\title{
3. Consumo del Cibo e Identità Locale nell'Antica Trattoria Arlati
}

Come accade quando si visita la casa di qualcuno, per entrare nella Trattoria Arlati bisogna suonare il campanello. Verso l'ora di pranzo, sostando per qualche minuto accanto al portone in ferro nero che fa da ingresso al locale, ci si può fare

\footnotetext{
${ }^{6} \mathrm{Nel} 2010$ la Trattoria Toscana da Aldo è entrata a far parte dell'albo delle Botteghe Storiche istituito dal Comune di Milano. 7 Utente Gianluca L (13 luglio 2011) http://www.yelp.it/biz/trattoria-toscana-da-aldo-milano (ultimo accesso: 30 ottobre 2013)

8 Le categorie di « cucina tradizionale », di «cucina casereccia» e di «cucina regionale» sono costruzioni culturali fortemente storicizzate nel territorio italiano. Per un'analisi approfondita dei processi storici che hanno portato a riempire di significato queste categorie si vedano, a titolo di esempio, i testi di Massimo Montanari (2004; 2010) o la raccolta di saggi curata de Mariani e Pirovano (2012). 9 Utente Steff (19 luglio 2006) http://www.2spaghi.it/ristoranti/lombardia/mi/milano/trattoria-toscana-da-aldo/ (ultimo accesso : 30 ottobre 2013) 
un'idea della clientela che frequenta questo luogo della commensalità: uomini in giacca e cravatta, donne in tailleur arrivano accompagnati da auto di lusso che ripartono subito dopo aver sostato qualche minuto all'incrocio tra via Alberto Nota e via San Glicerio. Come si legge sul sito ufficiale, la storia della Trattoria Arlati inizia negli anni '30 del Novecento quando Luigi e Modesta Arlati abbandonano il lavoro in Pirelli per aprire un'attività in proprio. Nel 1947 la sede della trattoria si sposta «in centro (della via, naturalmente!» e dall'angolo tra viale Sarca e via San Glicerio (a pochi passi dall'ingresso principale della Pirelli) si trasferisce in via Nota, sua sede attuale.

\begin{abstract}
«Passano cosi gli anni: tutti i giorni il tram e il trenino della Brianza scaricano alla Bicocca migliaia di lavoratori che alle sei di mattina si scaldano con tazze di grigioverde e bianchin sprusà ${ }^{10}$. Alle 5 di mattina Modesta è già in cucina a mettere su il brodo, base di tutti i piatti della cucina milanese e poi nervetti, risotti, stracotti, ossibuchi, minestrone cotolette e vitel tonnè. Intanto i figli grandi studiano e si laureano; Mario invece con il suo temperamento artistico vuole fare della trattoria la sua prima opera d'arte. Quando, alla fine degli anni '60 inizia a subentrare ai genitori lo fa trasformando il locale da osteria di periferia a ristorante frequentato da tutto il mondo artistico culturale della città attirato li dall'ambiente pieno di novità: l'arredamento un po' d'antiquariato e un po' bric e brac, l'atmosfera rilassata e cordiale, la cucina tradizionale ma sempre curata con una continua ricerca dell'ingrediente genuino, della ricetta da riscoprire». ${ }^{11}$
\end{abstract}

Entrando nel locale, accolti dal personale di sala, lo sguardo si posa su un enorme struzzo costruito con frammenti di carte da gioco, una delle tante opere d'arte che arredano le sale. Gli alti soffitti, le pareti di colore nero e l'illuminazione tenue sembrano voler creare un ambiente riservato e silenzioso. Appena varcata la soglia ci si ritrova in una piccola sala in cui, solitamente, vengono ricevuti i clienti; un vecchio armadio di legno sulla sinistra raccoglie i soprabiti mentre poco più avanti, sulla destra, un tavolo rotondo riunisce i membri della famiglia Arlati che, spesso, si incontrano a pranzo nella trattoria di famiglia. II locale è costituito da tre stanze al piano superiore e da un ambiente unico sul livello inferiore. II personale di servizio accompagna i clienti a dei tavoli coperti da tovaglie di cotone bianco, li fa accomodare su sedie imbottite i cui tessuti richiamano i colori delle pareti e delle tovaglie e consegna loro un menù la cui copertina è costituita da un'immagine dell'edificio che ospitava la prima sede del locale.

L'utente del portale web Tripadvisor 001Fab descrive l'ambiente di Arlati come una sorta di materializzazione delle storie delle persone che hanno trascorso del tempo nella trattoria:

«Arlati è un ristorante difficilmente descrivibile e identificabile. L'ambiente è il risultato di stratificazioni di oggetti e storie che con il passare degli anni hanno lasciato il loro contributo allinterno del locale. Originale, a tratti bizzarro, custodisce ricordi e ambientazioni meravigliosamente uniche nel loro genere. Le stanze con le pareti verniciate di nero illuminate dalla leggera luce di candele e pochissime lampade, i decori a tratti sontuosi a tratti ironici, la frammentazione degli spazi simile a quella di un appartamento, l'elegante professionalità ed il calore mai sfacciato del personale, $i$ cibi semplici ma gustosi ed un'aria lievemente decadente rendono questo gioiello milanese un indirizzo irrinunciabile per chi voglia provare un'esperienza unica nel suo genere. Chi come me ha avuto il piacere di frequentarlo varie volte non può non notare come sia totalmente diverso da tutto e tutti, come una serata al suo interno divenga ogni volta speciale e memorabile. I prezzi, più che onesti paragonati al tutto, perdono di importanza, mentre I'unica cosa che conta è ritornarci quanto prima». 12

Da questo punto di vista sembra che, contrariamente a quanto avviene per la Trattoria Toscana da Aldo, la Trattoria Arlati, pur rimanendo ubicata nel quartiere Bicocca, sia riuscita nell'impresa di trasformarsi insieme al quartiere stesso e a connettersi, anche dal punto di vista delle pratiche culinarie e dell'affluenza della clientela, a un discorso cittadino di più ampio respiro. In questo senso se Aldo si autorappresenta come prodotto del quartiere che tende ad autoconservarsi riattualizzando discorsi sulla memoria operaia che non sembrano più trovare luogo nel quartiere stesso, Arlati invece si vuole come un prodotto della città di Milano, come un luogo in cui la ricerca dei piatti e delle materie prime che, tradizionalmente, costituiscono la cucina lombarda trovano nelle modalità di preparazione dei piatti un sapere antico e tramandato di generazione in generazione.

Continuando a scorrere il sito ufficiale della Trattoria Arlati si legge:

\footnotetext{
10 /l grigioverde è un cocktail a base di grappa e sciroppo di menta; il "bianchin sprusà" (bianco sporco) è una bevanda alcolica a base di vino bianco frizzante e Campari.

11 http://www.trattoriaarlati.it/home/chi_siamo.html (ultimo accesso: 30 ottobre 2013).

12 Utente 001Fab (23 aprile 2012) http://www.tripadvisor.it/Restaurant_Review-g187849-d1052123-Reviews-or130-Trattoria_ArlatiMilan_Lombardy.htmI\#REVIEWS (ultimo accesso: 30 ottobre 2013)
} 
«Con il passare degli anni la città è cambiata; la Bicocca soprattutto è diventata il nuovo polo direzionale e universitario a Milano e anche la trattoria continua a cambiare: ai suoi tavoli manager e professori siedono al fianco di artisti, attori e cantanti. Nella gestione a Mario è succeduto il figlio Leopoldo che continua il lavoro del padre con freschezza ed entusiasmo. Lo studio e la ricerca nella cucina milanese e lombarda che in Mario poteva essere dettata dalla gola e dall'attaccamento alle tradizioni, in Leopoldo diventa premessa indispensabile per poter fornire un servizio adeguato ai giorni nostri, conservando però il calore della tradizione e il sapore dell'ingrediente genuino» ${ }^{13}$.

La continuità delle generazioni e delle tradizioni della famiglia Arlati e le trasformazioni che hanno interessato il quartiere Bicocca anche dal punto di vista dei collegamenti con il centro della città vengono ben sottolineate dalla recensione dell'utente di Tripadvisor Lucapaz che il 19 maggio 2013 ha scritto:

"Se la trattoria Arlati ha una tradizione di oltre 70 anni posso dire di esserne fortunato testimone da oltre 40..... Negli anni 70' andare a cena "da Mario" fino laggiù alla Bicocca...era quasi un viaggio fuori porta ma già ne valeva la pena per il cibo ma soprattutto per l'atmosfera. Gli arredamenti i dettagli e il sottofondo musicale facevano già intendere che Mario era un artista.... E stato bello recentemente scoprire che tutto è stato mantenuto grazie a Leo che è il figlio di Mario. II menù e l'atmosfera sono gli elementi più importanti nel giudizio di un ristorante. Leo ha creato un tocco in più con una cantina davvero interessante. La tradizione continua....».14

Arlati presenta la sua Trattoria come un locale della periferia diffusa che trova i suoi spazi di autorappresentazione sia nel quartiere e nella città sia negli spazi virtuali del proprio sito ufficiale e della Comunità di utenti di Tripadvisor sia attraverso un video postato su Yotube in cui Leopoldo Arlati, sulle note di Mo' Better Blues, presenta il locale della sua famiglia ponendo l'accento sui piatti milanesi e lombardi e sulla loro preparazione, terminando il discorso con la promessa: «Venendo in trattoria, nelle nostre stanze, sarete trattati come se foste a casa vostra» ${ }^{15}$ cercando così di includere gli avventori in un discorso che rimanda alle dimensioni famigliari e autentiche del consumo del cibo.

Da questo punto di vista è interessante notare che a molte delle recensioni lasciate dagli utenti corrisponde un commento 0 una risposta dell'utente Leo $\mathrm{A}^{16}$ (nickname di Leopoldo Arlati), che cerca in questo modo di creare un dialogo con i clienti che oltrepassi il tempo passato all'interno del locale. In questo senso, alla commensalità che il locale crea si cerca di fabbricarne anche un'altra costruita con i commenti e le critiche rispetto alle scelte dei cibi presentati, al tipo di servizio che viene offerto in sala che, a mio avviso, veicola in modo calzante un certo tipo di retorica sul consumo del cibo costruita sul costante richiamo alla ricerca di una identità milanese attraverso la memoria dei piatti tipici della città. Come si legge nella recensione di Marco P comparsa su Tripadivsor il 10 ottobre 2013:

«Trattoria Arlati ha una lunga tradizione, fu fondato negli anni '30 da una famiglia milanese, e ha mantenuto la sua tradizione culinaria, mentre saper adattare alle esigenze del cliente di oggi. Qui potrete godere di tutta la tradizione e cucina di Milano. L'atmosfera è gentile e cortese, ma anche molto professionale. Questo è davvero un ristorante dove il cliente è al centro dell'attenzione. Tipici piatti sono preparati con cura e abilità, mi dicono la lista dei vini è di buona scelta (...) - II ristorante si trova nei pressi della Bicocca, ora un'università e area business nonché residenziale. Assolutamente consigliato per le coppie in cerca di un'elegante cena squisita, per gli amici e per riunioni d'affari (il ristorante non è molto grande, suggerisco che per piccoli gruppi, non più di 10)».17

Inoltre, di particolare interesse è uno scambio di battute tra il proprietario e un avventore, autore di una recensione non troppo positiva; l'utente di Tripadvisor il-vero-Labo ${ }^{18}$ criticava l'accostamento del risotto al salto con l'osso buco e il fatto che la cotoletta alla milanese fosse troppo sottile, nella risposta di Leo A, il ristoratore legittima la sua scelta attraverso il riferimento a un discorso di autenticità supportato dal rimando alla tradizione regionale dei piatti proposti e dal riferimento alla memoria di famiglia che vuole che la nonna e la nonna della nonna fossero solite preparare gli stessi

${ }^{13}$ http://www.trattoriaarlati.it/home/chi_siamo.html (ultimo accesso: 30 ottobre 2013).

14 Utente Lucapaz (19 maggio 2013) http://www.tripadvisor.it/Restaurant_Review-g187849-d1052123-Reviews-or20-Trattoria_ArlatiMilan_Lombardy.htmI\#REVIEWS (ultimo accesso: 30 ottobre 2013)

15 http.//www.youtube.com/watch?v=U-yzvHMRMCU minuto $1: 30$ di 2 :14 (ultimo accesso: 30 ottobre 2013)

${ }^{16}$ Ad oggi l'Antica Trattoria Arlati ha ricevuto più di 180 recensioni sul portale web Tripadvisor.

17 Utente Marco P (10 ottobre 2013) http://www.tripadvisor.it/Restaurant_Review-g187849-d1052123-Reviews-or160-Trattoria_ArlatiMilan_Lombardy.htmIIREVIEWS (ultimo accesso: 30 ottobre 2013)

18 http://www.tripadvisor.it/Restaurant_Review-g187849-d1052123-Reviews-Trattoria_ArlatiMilan_Lombardy.html (ultimo accesso: 30 ottobre 2013) 
piatti nel medesimo modo19. In questo senso, posso affermare che la Trattoria Arlati posizioni la rappresentazione della famiglia come emblema della memoria culinaria regionale.

\section{Conclusioni}

Ho provato a fornire alcuni spunti di riflessione sulle retoriche costruite online e veicolate dalle pratiche di commensalità e di consumo del cibo nella trattoria Toscana da Aldo e nella Trattoria Arlati del quartiere Bicocca di Milano. Ho considerato come "nuovi abitanti" del quartiere coloro i quali frequentano la Bicocca per motivi di studio, di lavoro così come le persone che rispondono all'offerta di intrattenimento del quartiere, pur non risiedendo stabilmente in Bicocca. Ho dunque indagato i discorsi che gli avventori delle due trattorie diffondono utilizzando alcune recensioni pubblicate su importanti portali web. L'analisi di queste narrazioni mostra come gli avventori della Trattoria Toscana da Aldo interpretino il consumare il cibo come una riattualizzazione di un'ideologia politica. Nella Trattoria Arlati, invece, i discorsi sulla memoria fanno riferimento a un'identità cittadina e regionale piuttosto che di quartiere e a una ricerca di radici attraverso il servizio e la preparazione dei piatti, saperi trasmessi da una memoria familiare la cui continuità è ben testimoniata dalle piastrelle della cucina che riportano una sorta di albero genealogico della famiglia Arlati.

Sia nella Trattoria Toscana da Aldo che nella Trattoria Arlati si nota come la costruzione delle retoriche legate al cibo e al suo consumo facciano appello a una certa idea di autenticità della "vecchia Milano". I numi tutelari di questi discorsi sul passato sono da una parte gli anziani avventori abituali di Aldo, custodi della memoria mitica della sinistra e del movimento operaio locale, dall'altra le memorie della famiglia Arlati che sembrano dialogare senza grossi contrasti nell'autorità che definisce ciò che è "autenticamente" locale e cosa non lo è.

In conclusione, gli avventori della Trattoria Toscana da Aldo partecipano e costruiscono una commensalità che si delinea attraverso la condivisione di alcuni topoi e valori attribuiti, e attribuibili, a quello che viene letto come un passato mitico delle forze della sinistra nazionale e internazionale: le fotografie, i ritratti, la divisione degli spazi, le grandi tavolate che creano legami durante il tempo del pranzo rappresentano alcune delle manifestazioni dell'atto politico che gli avventori di Aldo attualizzano in questo luogo della commensalità. Nella Trattoria Arlati, invece, gli avventori sembrano costruire gli spazi della commensalità attraverso la condivisione di un'identità riconosciuta come tipica della città di Milano ${ }^{20}$; le retoriche che gli avventori costruiscono nei discorsi online mettono bene in evidenza come il consumo dei piatti proposti dalla Trattoria Arlati crei una connessione tra l'esperienza del consumo del cibo e l'appartenenza o la scoperta di questi tratti dell'identità. Le recensioni online di questi ultimi anni, inoltre, sottolineano come la costruzione degli spazi del quartiere e le relazioni sociali che il consumo del cibo crea trascendono sia lo spazio geografico del quartiere stesso sia quello storico in senso stretto. Queste fonti, tuttavia, riescono a creare altri modi di consumare il cibo, di fruire di un certo tipo di discorso sulla memoria e, contemporaneamente, a fabbricarne di nuovi. Più in generale, l'analisi comparativa di questi due casi etnografici mostra come la commensalità, manifestata e resa pubblica attraverso le recensioni online, lo scambio di informazioni e la narrazione delle proprie esperienze via web, definisca l'identità delle persone che, scegliendo cosa mangiare e dove farlo, dicono qualcosa di loro stessi. Infine, questo breve lavoro suggerisce che l'analisi etnografica di un contesto urbano come il quartiere Bicocca debba prendere in considerazione anche le interpretazioni, le rappresentazioni, le modalità con cui, attraverso la rete, vengono intrecciati discorsi di memoria della contemporaneità.

\section{Immagini}

Figura 1. Alcune fotografie tratte dal blog della Trattoria toscana da Aldo

\footnotetext{
${ }^{19}$ Nella risposta del 14 ottobre 2013 di Leo A all'utente II-vero-Labo si legge: "Pensi che quando ero bambino mia nonna, che è stata la prima cuoca della trattoria, mi faceva la cotoletta cosi' bassa e mi diceva sempre che era stata a sua volta sua nonna che le aveva insegnato a farla cosi'. Credo che come tradizione nostra di famiglia andiamo indietro di più di 120 annis.

${ }^{20}$ Nella lunga recensione di Patrizia L (13 gennaio 2013), tra l'altro, si legge : "La Trattoria promette e mantiene la cucina tipica milanese, dove per tipico si intendono i sapori, le composizioni, gli accostamenti, nulla a che vedere con il "tipico" che sottintende spesso il casereccio buono ma poco curato» (ultimo accesso: 30 ottobre 2013).

http://www.tripadvisor.it/ShowUserReviews-g187849-d1052123-r149681782-Trattoria_Arlati-Milan_Lombardy.html (ultimo accesso 30 ottobre 2013). 

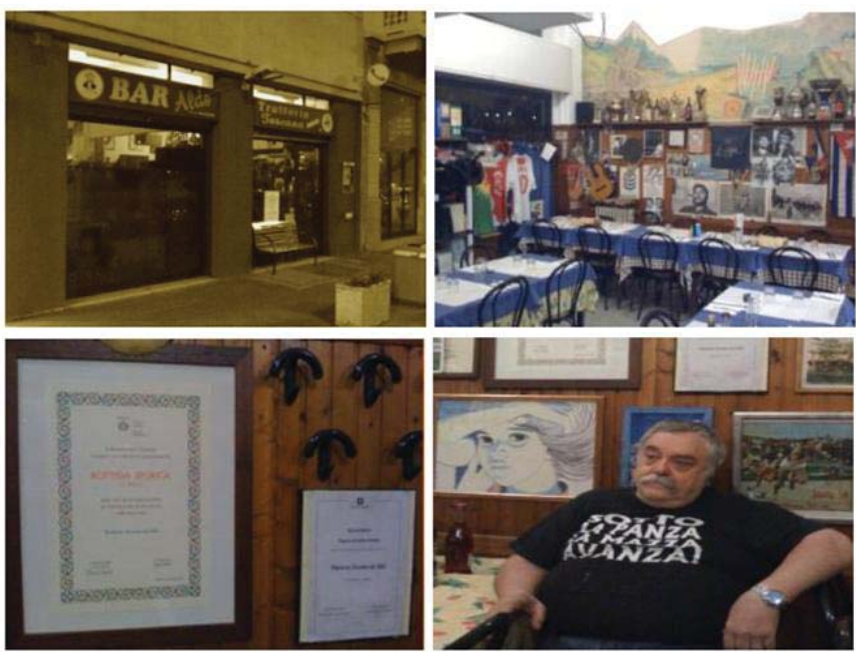

http://www.trattoriatoscanadaaldo.restaurant.point.in.citylandia.org

Figura 2. Alcune fotografie tratte dal sito ufficiale della Trattoria Arlati
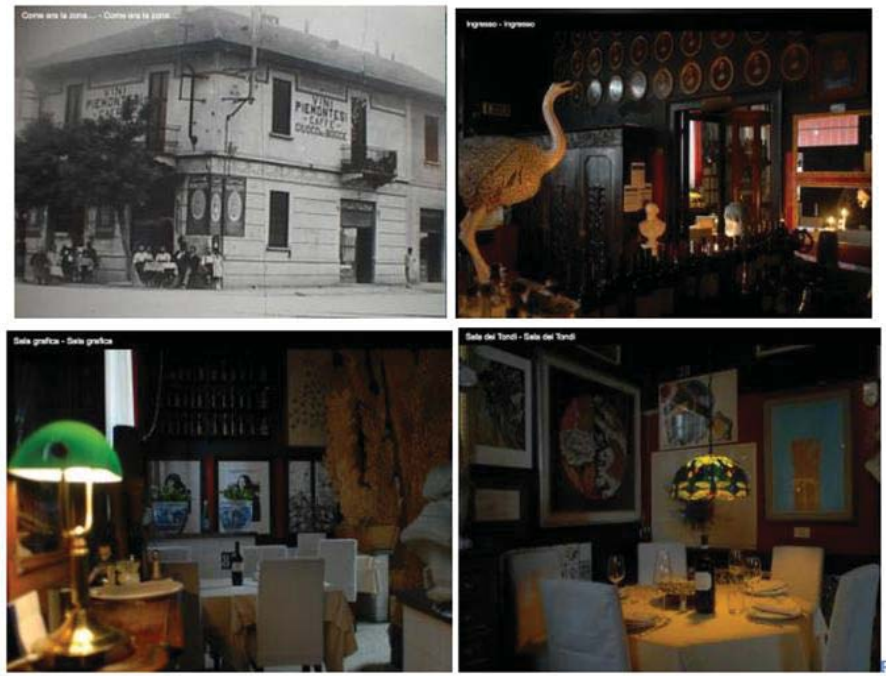

http://www.trattoriaarlati.it/gallery/

\section{Bibliografia}

Berliner, D., (2005) Social Though \& Commentary: The Abuses of Memory: Reflections on the Memory Boom in Anthropology. Anthropological Quarterly Vol. 78 (1) pp. 197-211

Carter, D., (2005). Living in Virtual Communities. An Ethnography of Human Relationship. Cyberspace, Information, Comunication and Society Vol. 8 (2) pp.148-167

Cercone, A., (2007). Dagli Stipiti il pranzo è servito. II Giorno, domenica 6 maggio 2007

Dei, F., (2004) Antropologia e memoria. Prospettive di un nuovo rapporto con la storia. Novecento Vol. 10 pp. 27-46

Esposito, R., (1988). Categorie dell'impolitico, II Mulino:Bologna

Fabietti, U.; Matera, V., (Eds.), (2000) Memoria e identità. Simboli e strategie del ricordo, Roma: Meltemi 
Hannerz, U., (1992). Esplorare le città. Antropologia della vita urbana, Bologna: II Mulino

Hine, C., (2000). Virtual Ethnography, New York :Sage

Holtzman, J.D., (2006). Food and Memory. Annual Review of Anthropology Vol. 35 pp. 361-378

Lévy P., (1997). II virtuale, Milano:Raffaello Cortina

Mariani, L.; Pirovano, M., a cura di (2012). Il cibo e gli uomini. L'alimentazione nelle collezioni etnografiche lombarde, Lecco: Rebel

Montanari, M., (2004). II cibo come cultura. Torino: Laterza

Montanari, M., (2010). L'identità italiana in cucina. Torino: Laterza

Murphy, P., (2011). Locating media ethnography. In V. Nightingale (Ed.). Handbook of Media Audiences. New York: Blackwell Publishing

Portelli, A., (1985). Biografia di una città. Storia e Racconto: Terni 1830-1985, Torino:Einaudi

Portelli, A., (2007). Storie orali. Racconto, immaginazione, dialogo, Roma, Donzelli

Rimoldi, L., (2012). The Construction and the Negotiation of Ethnographic Voices Notes From an Italian Post-Industrial Area. Mediterranean Journal of Social Sciences Vol. 3 (8) pp. 221-226

Rimoldi, L., (2013). «The Kitchen of the Revolution». Food and Working-class Memory in the Bicocca Area (Milan, Italy). Mediterranean Journal of Social Sciences, Vol 4 (3) pp. 778-785

Sutton, D.E., (2001). Remembrance of Repasts: An Anthropology of Food and Memory, Oxford: Berg

\section{Siti internet consultati}

http://www.yelp.it

http://www.2spaghi.it

http://www.tripadvisor.it

http://www.trattoriatoscanadaaldo.restaurant.point.in.citylandia.org

http://www.trattoriaarlati.it

http://www.youtube.com 
